\title{
Lisdexamfetamine in the treatment of moderate-
} to-severe binge eating disorder in adults:

\section{systematic review and exploratory meta-analysis of publicly available placebo-controlled, randomized clinical trials}

This article was published in the following Dove Press journal:

Neuropsychiatric Disease and Treatment

25 July 2016

Number of times this article has been viewed

Michele Fornaro, ${ }^{1,2}$ Marco

Solmi, ${ }^{3-5}$ Giampaolo Perna, ${ }^{2,6}$

Domenico De Berardis, 2,7

Nicola Veronese, ${ }^{5,8}$ Laura

Orsolini, ${ }^{2,9}$ Licinia Ganança, ${ }^{1,10}$

Brendon Stubbs ${ }^{11,12}$

'New York State Psychiatric Institute, Columbia University, New York City, NY, USA; 'Polyedra Research Group ${ }^{\oplus}$, Ascoli, ${ }^{3}$ Department of Neurosciences, University of Padua, ${ }^{4}$ Department of Mental Health, National Health Service, Padova IIREM Institute for Clinical Research and Education in Medicine, Padova, ${ }^{6}$ Department of Clinical Neurosciences, Hermanas Hospitalarias - Villa San Benedetto Menni Hospital, FoRiPsi, Albese con Cassano, Como, ${ }^{7}$ Department of Mental Health, Psychiatric Service of Diagnosis and Treatment, National Health Service, Hospital “G Mazzini”, Teramo, ${ }^{8}$ Department of Medicine (DIMED), University of Padua, Padova, Italy; ${ }^{\text {Psychopharmacology, Drug }}$ Misuse and Novel Psychoactive Substances Research Unit, School of Life and Medical Sciences, University of Hertfordshire, Hatfield, Herts, UK; ${ }^{10}$ Department of Psychiatry, School of Medicine, University of Lisbon, Lisbon, Portugal; "Department of Health Service and Population Research, Institute of Psychiatry, King's College London, ${ }^{12}$ Department of Physiotherapy, South London and Maudsley NHS Foundation Trust, London, UK

Correspondence: Michele Fornaro New York State Psychiatric Institute, Columbia University, 105I Riverside Drive, Unit 42, room 2729, New York City 10032, NY, USA

Tel +l 6467745000

Email dott.fornaro@gmail.com
Background: Preliminary placebo-controlled evidence paved the ground to the US Food and Drug Administration approval extension of lisdexamfetamine for the treatment of moderateto-severe binge eating disorder (BED) in adults.

Objectives: To provide a preliminary qualitative and quantitative synthesis of the placebocontrolled, randomized clinical trials (RCTs) considering the efficacy and tolerability of lisdexamfetamine in the acute and/or maintenance treatment of moderate-to-severe BED in adults.

Methods: A preliminary, yet comprehensive, systematic review was performed by accessing a broad range of resources providing publicly available data about lisdexamfetamine at the time of inquiry (March 2016). Study eligibility criteria, participants, and interventions were considered focusing on major clinical and functional outcomes of either efficacy or tolerability of lisdexamfetamine in the treatment of moderate-to-severe BED in adults.

Results: Meta-analysis of data pooled from three acute RCTs significantly favored lisdexamfetamine over placebo in the reduction of binge eating days/week, Yale-Brown Obsessive Compulsive Scale Modified for Binge Eating total score, weight, response, and remission rates (all, $P \leq 0.01$ ). In contrast, discontinuation rates due to treatment-emergent adverse events were significantly higher among patients in receipt of lisdexamfetamine (relative risk 2.19, $P=0.04$ ) versus placebo.

Limitations: Publication, selection, performance, attrition, reporting, sponsorship, and "diagnostic shift" biases. Lack of inclusion of adverse event effects other than those requiring discontinuation of the trial(s), as well as lack of information about clinically relevant psychiatric or other medical comorbidities, limits the overall generalizability of pooled results.

Conclusion: Across the included acute phase RCTs, lisdexamfetamine (at 30, 50, or $70 \mathrm{mg}$ /day) led to significant reduction in a number of clinically relevant outcomes compared to placebo. Moreover, safety concerns related to adverse events, high discontinuation rates, and the need for additional long-term maintenance of RCTs solicit careful monitoring of the drug in terms of overall safety and tolerability by further RCTs.

Keywords: lisdexamfetamine, binge eating disorder, systematic review, meta-analysis

\section{Introduction}

Already enlisted by the Diagnostic and Statistical Manual for Mental Disorder Fourth Edition (DSM-IV) ${ }^{1}$ and its Text-Revision $(D S M-I V-T R)^{2}$ as a provisional (“appendix B”) 
category of "eating disorder (ED) not otherwise specified", binge eating disorder (BED) is characterized by recurrent episodes of binge eating occurring in a discrete period of time ( $\leq 2$ hours), involving a sense of lack of control over the consumption of an amount of food that is definitely larger than most people would consume under similar circumstances. Lack of control over eating is a core feature of BED distinguishing it from overeating. ${ }^{3}$

BED lifetime prevalence among US adults approximates $2.6 \%$, which is higher than the one documented for anorexia nervosa and bulimia nervosa combined together, though sex distribution of BED is less skewed in comparison. ${ }^{4,5}$ In addition, while no racial group is significantly overrepresented, ${ }^{6} \mathrm{BED}$ is traditionally documented to be more common among young obese individuals, who usually receive first diagnosis of BED early in life around mid-1920s, ${ }^{7,8}$ although no minimum age threshold has been established for the diagnosis. ${ }^{9}$

Higher prevalence rates of BED would nonetheless be expected, both in $\mathrm{UK}^{10}$ and US community ${ }^{11}$ and clinical ${ }^{11}$ samples. These higher figures would ultimately reflect a higher diagnostic sensitivity developed by the clinicians over the time, as well as the impact of more permissive criteria introduced by the Fifth Edition of the DSM (DSM-5), ${ }^{9}$ finally acknowledging BED as a distinct diagnostic category now characterized by reduced "frequency" ("once per week" instead of "twice per week") and "duration" ("three months" vs "six months") criteria compared to the DSM-IV ones. ${ }^{9,12,13}$

Many cases of BED may still go underdiagnosed for many years because patients seeking psychiatric treatment for either psychiatric or somatic disorders are not always specifically asked about their eating behavior. ${ }^{14}$ Earlier detection and optimal multidisciplinary, stepped-care approach to BED nonetheless represent primary needs, with a special emphasis toward a better pharmacological management. ${ }^{11,15-17}$

In this regard, many of the available pharmacological studies on BED have long been plagued by limitations such as small sample size, high placebo response and/or dropout rates, and limited generalizability of findings to real-world practice, ${ }^{18,19}$ with no conclusive advantage documented for any drug over alternative approaches, especially dietary, or cognitive behavioral therapy, just to name few. ${ }^{19}$

This is compelling, especially considering that various neurobiological underpinnings of BED primarily focusing on dopamine, opioid, acetylcholine, and serotonin neurocircuitry within brain reward regions have been advocated to play a role on the matter in both animal and human studies. ${ }^{19,20}$
To date, evaluated agents targeting neurotransmitter systems allegedly involved in motivated feeding behavior, mood regulation, and impulse control include several antidepressant and anticonvulsant agents that demonstrated efficacy in reducing binge eating frequency, but only in limited cases these effects have resulted in patients achieving abstinence, which would be the primary goal of the treatment; they also range from less (fluvoxamine) to more (topiramate) effectiveness in achieving weight loss, though clinically meaningful and significantly greater than placebo for both drugs. ${ }^{19}$

Moreover, many individuals with BED receive pharmacotherapy ${ }^{21,22}$ in the absence of any US Food and Drug Administration (FDA)-approved drug, ${ }^{3,23}$ at least until early 2015, when the central nervous system (CNS)stimulant lisdexamfetamine dimesylate (hereafter referred to as "lisdexamfetamine") was granted extended approval for the treatment of moderate-to-severe cases of BED in adults - but weight loss - beyond the 2007 sole approval of attention deficit hyperactivity disorder (ADHD). ${ }^{24}$

The present systematic review and exploratory metaanalysis thus evaluated preliminary placebo-controlled evidence about the efficacy and safety of lisdexamfetamine in moderate-to-severe adult cases of BED.

\section{Methods}

\section{Data source and search methods}

The present systematic review adhered to the Preferred Reporting Items for Systematic reviews and Meta-Analyses (http://www.prisma-statement.org/), ${ }^{25}$ while meta-analysis adhered to the Meta-analysis Of Observational Studies in Epidemiology guidelines. ${ }^{26}$ We searched for Englishlanguage published randomized clinical trial (RCTs) comparing lisdexamfetamine versus placebo for the treatment of moderate-to-severe BED in adults (both sexes). Eventual adjunctive therapies of lisdexamfetamine with any additional drug, physical therapy, or psychotherapy were also taken into account. Although our search strategy accounted for results indexed from inception until the writing time (March 2016), special emphasis was placed by two appointed authors (MF and LO) on those results indexed since January 2008, as these latter would reflect the recent DSM-5's introduction of BED as an autonomous disorder, following hierarchical considerations ${ }^{17}$ and the notion that articles claiming BED inclusion in DSM-5 dated back to that time. ${ }^{27}$

The US National Library of Medicine's PubMed resource (http://www.ncbi.nlm.nih.gov/pubmed/), the US ClinicalTrials. gov registry (https://ClinicalTrials.gov/), the EU Clinical Trials 
Register (https://www.clinicaltrialsregister.eu/), the Web of Science $^{\circledR}$ (Thomson Reuters $\left.{ }^{\circledR}\right)$, Scopus (Elsevier's ${ }^{\circledR}$ ) (http:// www.scopus.com/), EMBASE ${ }^{\circledR}$ (Elsevier's $\left.{ }^{\circledR}\right)$, CINAHL $^{\circledR}$ (EBSCO Nursing Resource ${ }^{\circledR}$ ) (https://www.ebscohost.com/ nursing/products/cinahl-databases/cinahl-complete), and PsycINFO $^{\circledR}$ (American Psychological Association) (http:// www.apa.org/pubs/databases/psycinfo/) databases were inquired on March 1, 2016. Only "completed" placebocontrolled RCTs with posted results were considered for inclusion in either the US or EU registers.

Publicly available press release materials, conference posters, and meeting abstracts posted at the website of the manufacturer of lisdexamfetamine $\left(\right.$ Shire $\left.^{\circledR}\right)$ were likewise accounted. The searched terms across different sources included: "lisdexamfetamine", "lisdexamphetamine", "SPD489" (Lisdexamfetamine dimesylate); "vyvanse" $\left({ }^{\circledR}\right)$, "binge eating disorder", "placebo-controlled clinical trial", "open-label", "moderate-to-severe", "adults", or their combination.

The adopted MEDLINE search string was: " $((()((()(()(($ lisdexamfetamine) AND binge eating disorder) AND clinical trial) OR lisdexamphetamine) AND binge eating disorder) AND clinical trial) OR SPD489) AND binge eating) AND clinical trial) OR lisdexamfetamine) AND binge eating disorder) AND moderate) AND severe) AND adults". Attempt to retrieve additional bibliographic references was made screening the reference list of the selected published studies and relevant review articles whenever available. We also planned into advance contact with the authors of proof-of-concept studies, Phase II, III, or IV RCTs if ever indexed, in order to gather additional information relevant to the research theme at study. The Cochrane Library (http://www.cochranelibrary. com/) was also accessed aiming at detecting unpublished or negative results, thus striving to avoid any "sponsorship bias" as much as possible, as performed by inquiring the Journal of Pharmaceutical Negative Results (http://www. pnrjournal.com/), the Journal of Negative Results - Ecology \& Evolutionary Biology (www.jnr-eeb.org), and the Journal of Negative Results in Biomedicine (www.jnrbm.com).

Finally, both auto- and hand-searches for "type-I" ("duplicates among/across different databases") and "type-II" ("duplicate publications in different Journals/issues) ${ }^{28}$ were performed using Review Manager v 5.3 ${ }^{\mathrm{TM}}$ for Microsoft Windows $^{\text {TM }}{ }^{29}$ Documents focusing (only) on children or adolescents, ADHD trials, case reports/series, or preclinical or otherwise incomplete or nonpublicly available data were excluded.

\section{Outcome measures}

Efficacy measures were "reduction in binge eating days/ week" from least square means with mixed effects model for repeated measures (MMRM) or from last observation carried forward, where MMRM data were not available, accounted as the primary outcome measure, while "reduction in the Yale-Brown Obsessive Compulsive Scale Modified for Binge Eating (YBOCS-BE) ${ }^{30}$ total score" was adopted as secondary efficacy measure.

Response rates across trials were defined by a Clinical Global Impressions (CGI) Improvement score ${ }^{31}$ of "very much improved" or "much improved". Remission was defined by 4 -week cessation of binge eating. Reduction in weight was also considered across documenting studies. Discontinuation rates because of treatment-emergent adverse events (TEAEs) were also regarded across reporting studies. The primary endpoint was "time to relapse of binge eating symptoms in adults with moderate-to-severe BED”. For maintenance studies, the primary outcome measure was "time to relapse of binge eating symptoms"; additional outcomes included "proportion of relapse vs placebo" at study endpoint, among other measures.

Additional qualitative results were recorded whenever documented across the included studies. Finally, though informative, positive predictive value and negative predictive value were not systematically recomputed nor presented herein as they were already extracted for most of the relevant $\mathrm{RCTs},{ }^{3}$ but the latest available maintenance $\mathrm{RCT}^{32}$ and the notion that such Bayesian index could be misleading when preferred to effect size computations using meta-analytic approaches. ${ }^{33,34}$

\section{Data analysis}

We decided "a priori" to analyze data using a random effect model due to heterogeneity in study duration, phase of the trial, primary outcome measures and clinical samples, or dose of the drug. A random effect model incorporates both withinand between-study variance into the estimate of average treatment effects and is therefore appropriate when analyzing studies with methodological heterogeneity. The standard mean differences (SMDs) for continuous variables and relative risk (RR) for dichotomous outcomes were calculated with $95 \%$ confidence interval (CI) limit. The analyses were conducted using the Comprehensive Meta-analysis ${ }^{\mathrm{TM}} \mathrm{V} 3$ for Microsoft Windows ${ }^{\mathrm{TM}}$ software (Englewood, NJ, USA). ${ }^{35}$ For those studies presenting results in the standard error of mean (SEM) form; standard deviation (SD) was converted to $\mathrm{SEM}$ using the formula: $\mathrm{SD}=\mathrm{SEM}^{*} \sqrt{ } \mathrm{n}$, where $\mathrm{n}$ is the 
sample size. Data were entered and/or extracted by three leading authors (MF, MS, and BS) assisted by additional authors (GP, DDB, LO, NV, and LG). Heterogeneity was assessed by the $\mathrm{I} 2$ statistic. ${ }^{36}$ For each pooled analysis, in order to test for publication bias, a trim and fill adjusted analysis was conducted $^{37}$ in order to remove the most extreme small studies from the negative or positive side of the funnel plot, and the effect size at each iteration was recalculated, until the funnel plot was symmetric about the (new) effect size.

\section{Results}

\section{Studies included in the analysis}

Details about the multistep screening of results have been outlined in Figure 1. Stating the very preliminary nature of the present systematic review and meta-analysis, only four original studies met our inclusion criteria, including one preliminary maintenance report, ${ }^{32}$ a Phase II RCT, ${ }^{38}$ and two pivotal Phase III RCTs for which results were presented in a single report. ${ }^{39}$ The included acute-treatment RCTs recruited different samples of participants. Table 1 provides the details of the studies.

\section{Qualitative synthesis of results}

The results of the only preliminary maintenance study were made available to the public through the manufacturer's press-release webpage. ${ }^{32}$ All the studies were multicenter trials sponsored by the drug manufacturer and conducted either in the USA or Germany (and Spain or Sweden for

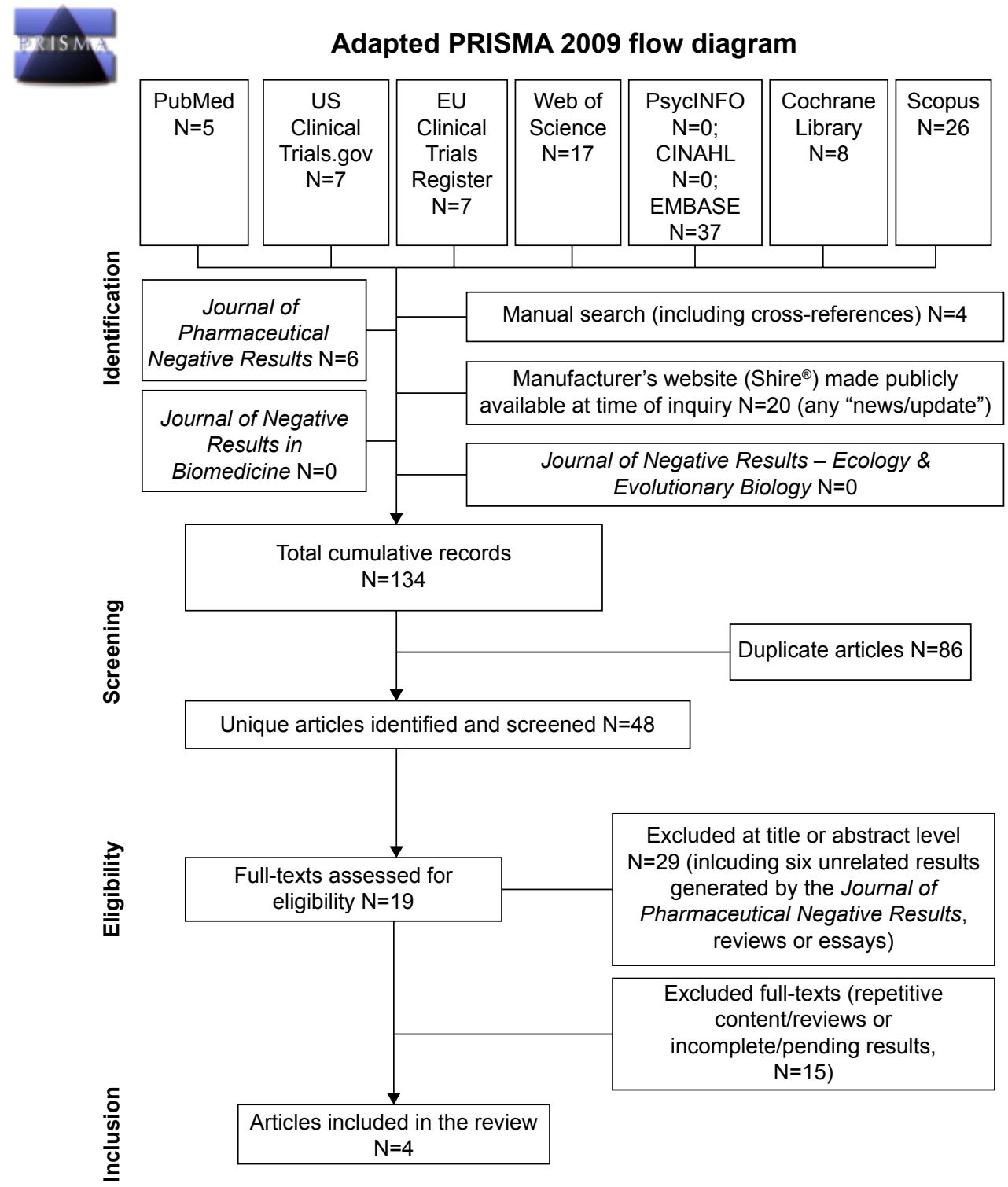

Figure I Flowchart of the included studies.

Notes: Adapted from Moher D, Liberati A, Tetzlaff J, Altman DG; PRISMA Group. Preferred reporting items for systematic reviews and meta-analyses: the PRISMA statement. PLoS Med. 2009;6(7):e 1000097.25 


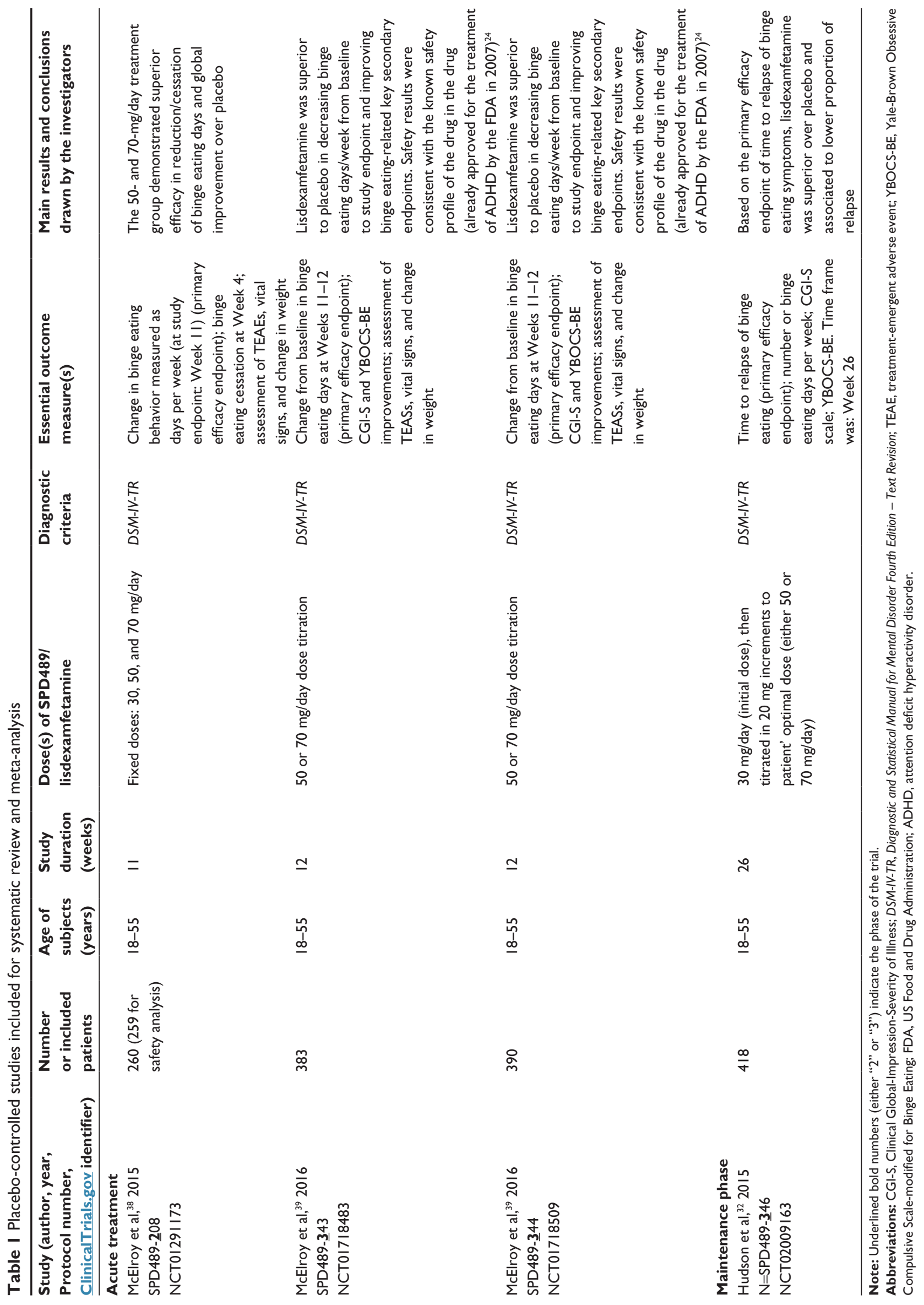


Phase III trials). Anorexia nervosa, bulimia nervosa, ADHD, as well as other relevant psychiatric comorbidities or medications were shared exclusion criteria. All included trials based on the $D S M-I V-T R^{2}$ criteria rather than the more permissive $D S M-5^{9}$ ones were made officially available after the registration of the studies.

Statistically significant reduction $(P \leq 0.01)$ in binge eating days/week (primary efficacy outcome across the included studies) were observed for lisdexamfetamine doses of 50 and $70 \mathrm{mg} /$ day with large effect sizes in the three acute RCTs. ${ }^{38,39}$ Similarly, lisdexamfetamine proved statistically significant $(P \leq 0.01)$ superiority over placebo in the maintenance trial with regard to the primary endpoint "time to relapse of binge eating symptoms". ${ }^{32}$ Specifically, in the 11-week Phase II trial (protocol number: SPD489-208), ${ }^{38}$ the difference for lisdexamfetamine versus placebo MMRM least square (LS) mean change from baseline to week 11 on log transformed binge eating days per week was (mean \pm SEM) -0.01 (0.096), $P=0.88 ;-0.26$ (0.096), $P=0.01 ;-0.35$ (0.096), $P<0.01$ for lisdexamfetamine doses of 30,50 , or $70 \mathrm{mg} /$ day, respectively, favoring lisdexamfetamine doses of 50 and $70 \mathrm{mg} /$ day but not of $30 \mathrm{mg} /$ day. In the acute-treatment 12-week trials (protocol numbers: SPD489-343 and SPD489-344), ${ }^{39}$ the difference for lisdexamfetamine versus placebo MMRM LS mean change from baseline to week 11 in binge eating days per week was $-1.35(1.7,-1.01)$, $P<0.01$ for study 1 (SPD489-343), with mean lisdexamfetamine dosage of $56.9+9.72 \mathrm{mg} /$ day, and $-1.66(-2.04$, 1.28), $P<0.01$ with mean lisdexamfetamine dosage of $57.6+9.24 \mathrm{mg} /$ day in study 2 (SPD489-344). ${ }^{39}$

Pooled (30, 50, or $70 \mathrm{mg} /$ day) "responders' rates" as defined by a CGI-S improvement score of "very much improved" or "much improved" in the acute trials versus placebo were as follows: $89.6 \%$ versus $64.5 \%{ }^{38}$ or $82.1 \%$ versus $47.3 \%$ (SPD489-343), ${ }^{39}$ or $86.2 \%$ versus $42.6 \%$ (SPD489-344), ${ }^{39}$ respectively.

Pooled "remission rates" versus placebo as defined by "4-week cessation of binge eating" in the acute-treatment trials were: $42.3 \%$ versus $21.3 \%^{38}$ or $40 \%$ versus $14.1 \%$ $(\mathrm{SPD} 489-343)^{39}$ or $36.2 \%$ versus $13.1 \%$ (SPD489-344), ${ }^{39}$ respectively.

Pooled "discontinuation rates" due to TEAEs were as follows: $3.6 \%$ versus $0 \%$ or $6.3 \%$ versus $2.7 \%$ (SPD $489-343)^{39}$ or $3.9 \%$ versus $2.7 \%$ (SPD489-344), ${ }^{39}$ respectively.

Any TEAE frequency was significantly higher in the lisdexamfetamine group compared with placebo; $84.7 \%$ versus
$58.7 \%$ (SPD489-208), ${ }^{38} 82.3 \%$ versus 58.8\% (SPD489343), ${ }^{39} 77.3 \%$ versus 50.8\% (SPD489-344). ${ }^{39}$ Moreover, one patient died due to methamphetamine overdose (in the SPD489-208 trial) though this event was considered unrelated to the trial itself, ${ }^{38,40}$ and severe adverse event (AE) frequency in the lisdexamfetamine group was equal to $8.9 \%$ versus $3.2 \%$ (SPD489-343), ${ }^{39}$ and $3.9 \%$ versus $3.2 \%$ (SPD489-344). ${ }^{39}$

Concerning the Phase-III, 26-week maintenance placebocontrolled RCT, ${ }^{32}$ lisdexamfetamine demonstrated superiority over placebo $(P \leq 0.01)$ with respect to the primary efficacy endpoint ("time to relapse"). Notably, the maintenance trial actually consisted of a 4-week screening period, a 12-week open-label treatment phase (4 weeks of dose optimization and 8 weeks of maintenance), followed by a 26 -week, doubleblind, randomized withdrawal phase and a follow-up visit 1 week after the last on-treatment visit. Overall, 418 patients were included in the study; yet exact figures of those allocated to either lisdexamfetamine (50 or $70 \mathrm{mg}$ /day) rather than placebo were not publicly disclosed at the writing time. Similarly, during the randomized withdrawal phase, two patients receiving lisdexamfetamine experienced serious AEs (undisclosed at the writing time). The most commonly reported TEAEs (reported by $5 \%$ or more of the patients) leading to study discontinuation were documented in the prerelease report available to the public; yet exact proportions were not disclosed at the writing time. Therefore, the quantitative extractions focused on the three acute-treatment trials, the results of which were made publicly available through two papers. ${ }^{38,39}$

\section{Meta-analysis}

Only the 11-week Phase II trial (protocol number: SPD489$208)^{38}$ reported separate results for 30,50 , and $70 \mathrm{mg} /$ day of lisdexamfetamine groups, which we analyzed together with data from the acute-treatment 12-week trials (protocol numbers: SPD489-343 and SPD489-344). ${ }^{39}$ Also, we could not include the maintenance trial in our meta-analysis since it had different primary outcomes ("time to relapse"). Thus, only three ${ }^{38,39}$ of four ${ }^{32,38,39}$ original studies included in the systematic review were also included in the quantitative analysis, as detailed in Table 2.

A total of 450 patients received placebo, and 583 patients were randomized to lisdexamfetamine, of whom 66, 65, and 65 patients were allocated to doses of 30, 50, and $70 \mathrm{mg} /$ day, respectively, versus 64 patients randomized to receive placebo in the 11-week Phase II trial. ${ }^{38}$ 


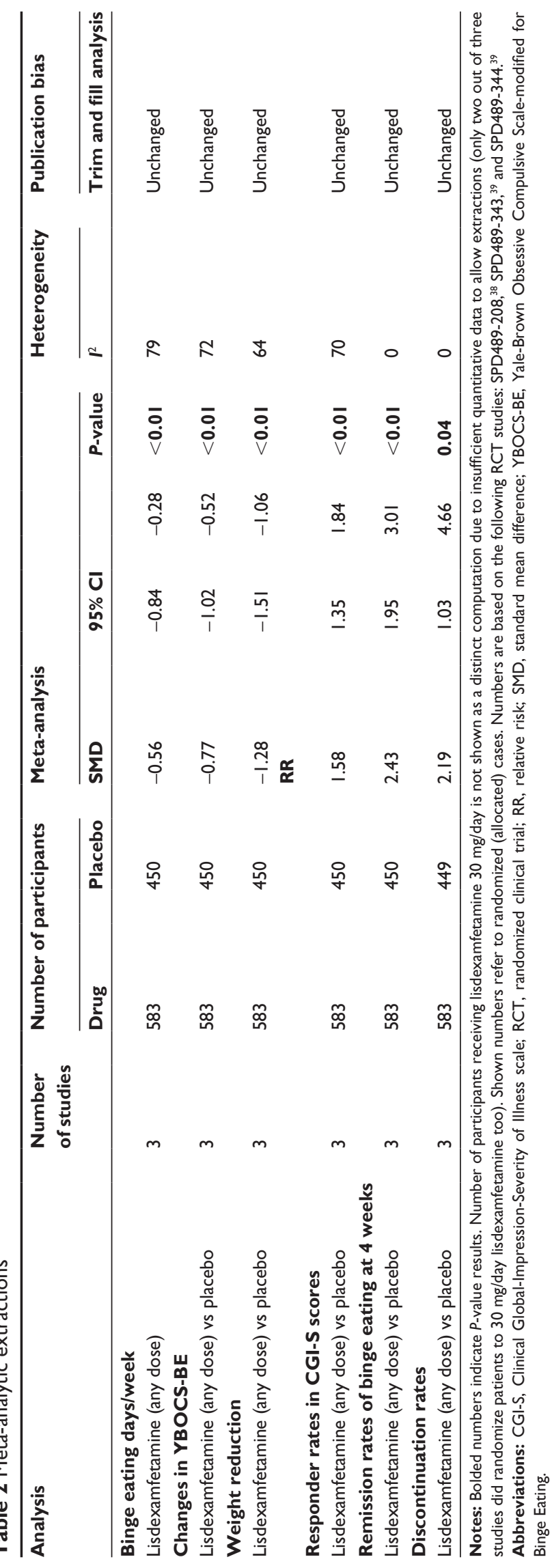

Primary efficacy outcome:"change in binge eating days/week at study endpoint" across different dosages of the drug versus placebo

Lisdexamfetamine reduced days with binge eating significantly more than placebo (studies $=3, \mathrm{SMD}=-0.56$ $[-0.84,-0.28], P<0.01)$.

Quantitative synthesis of results:"change in CGI-S, YBOCS-BE scores, remission rates ('4-week cessation of binge eating') and mean weight reduction, in $\mathrm{kg}$ "

Change in YBOCS-BE scores statistically significantly differed among patients receiving lisdexamfetamine (any dose) versus placebo $(P \leq 0.01)$ in single studies and meta-analyzing all data together (studies $=3, \mathrm{SMD}=-0.77$ $[-1.02,-0.52], P<0.01)$.

Comparisons of lisdexamfetamine (any dose) versus placebo showed significantly higher rates of response based on the CGI-S scores (studies $=3, \mathrm{RR}=1.58[1.35,1.84], P<0.01$ ) and of remission (4-week binge cessation) (studies $=3$; $\mathrm{RR}=2.43[1.95,3.01], P<0.01)$. Mean weight reduction was significantly higher in lisdexamfetamine group compared with placebo (studies $=3, \mathrm{SMD}=-1.28[-1.51,-1.06], P<0.01$ ).

Quantitative synthesis of results: "discontinuation rates due to TEAEs"

Discontinuation rates among patients receiving lisdexamfetamine - any dose - were significantly higher versus placebo ( studies =3, RR =2.19 [1.03, 4.66], $P=0.04$ ).

\section{Discussion}

The present study has suggested some early and promising evidence supporting the efficacy of lisdexamfetamine, a cost-effective treatment for moderate-to-severe BED adults in the USA. ${ }^{41}$ This encouraging evidence was consistent across all the primary and secondary outcomes accounted in the included acute-trials (meta-analysis) and also the maintenance trial (systematic review). However, TEAEs and subsequent discontinuation rates were significantly higher among those patients receiving lisdexamfetamine.

\section{Main results and major implications for the clinical practice}

Overall, the days with binge behavior, the response, and remission rates indicate solid and consistent efficacy profile 
of lisdexamfetamine in BED; however, these results should be considered as preliminary. Moreover, the Y-BOCS-BE scale provides an accurate measuring of the binge eating behavior. A significant reduction in its total scores for those patients allocated to 50 or $70 \mathrm{mg}$ /day lisdexamfetamine rather than placebo is therefore clinically meaningful. Similarly, since most bingers might be overweight patients, the occurrence of a significant weight reduction with lisdexamfetamine versus placebo is also clincially relevant, although it must be remarked that lisdexamfetamine is not FDA-approved for weight reduction/control at the writing time and that overweight or frank obesity is not included among the DSM-5 codes.

This latter consideration has further major clinical implications, especially considering that CNS stimulants may be abused by overweight people, regardless the presence of fullthreshold binge eating behavior ${ }^{42-44}$ and that, to some extent, weight loss would be a side effect for some other patients rather than a treatment benefit. The presence of cardiometabolic illness further increases the risk of pulmonary hypertension among people receiving CNS stimulants. ${ }^{45}$ Insomnia is also a common side effect associated with CNS stimulants. ${ }^{46}$ While the AEs documented in the studies included in the present systematic review (three acute-treatment trials and one maintenance RCT) were not life-threating ones, not even leading to trial discontinuation, additional investigations about the acute- and maintenance-treatment safety and tolerability of lisdexamfetamine are warranted, despite preliminary evidence on the matter would be consistent with the overall safety profile documented among ADHD patients exposed to comparable doses of the drugs (at least in the acute setting). Long-term/maintenance trials are definitely needed (one ongoing trial at the writing time: NCT01657019). This is not just with reference to placebo comparisons, but also versus other drugs often prescribed as off-label treatment of moderate-to-severe BED in adults, including the pro-drug methylphenidate as well as the non-CNS stimulant antidepressant drugs (ie, fluoxetine or bupropion).

Finally our results bring attention on safety aspects of lisdexamfetamine, which should be carefully evaluated by further long-term studies, ideally including also adolescent and old age subjects. ${ }^{40}$ In addition, studies conducted by independent researchers and not funded by the manufacturer would help reduce any potential bias.

\section{Limitations of the study}

Though informative, the present review and meta-analysis only included four original studies, of which only three were suitable for pooled quantitative extraction. Nonetheless, it must be remarked that as little as two studies should be acceptable for a preliminary meta-analytic report, even when accounting for heterogeneous active compounds in the same class of drugs and/or differential doses, ${ }^{47}$ whereas those meta-analysis accounting for substantially heterogeneous compounds and/or based on incomplete sources would not be informative, not even if including a high number of studies. ${ }^{48}$ Moreover, we acknowledge that meta-analyses with $<20$ studies have limited power to detect publication bias. ${ }^{49}$

Specifically, the present systematic review actually included three homogenous acute-treatment studies and one additional maintenance study. Yet, only selected outcomes/ moderators documented for the acute-treatment trials could be pooled together since both the operational criteria and exact prevalence rates of efficacy or safety measures in the maintenance phase were either undisclosed at the writing time or presented as a mean of the double-blind plus the open-label phase. Inclusion of pooled number obtained by open-label phases too were thus avoided in order to ensure consistency of quality of the pooled results.

Concerning additional potential biases, the acknowledgment of BED as a full diagnostic category by the DSM-5 and greater attention by clinicians toward the burden associated with BED most likely also lead to increased attention of the brands toward the pharmacological management of such condition. Nonetheless, the FDA extended the approval of lisdexamfetamine also for the treatment of moderate-to-severe BED in adults ${ }^{24}$ based on DSM-IV-TR ${ }^{2}$ rather than the more permissive $D S M-5^{9}$ criteria. Moreover, the FDA extended approval based on two Phase III studies ${ }^{39}$ and one Phase II study ${ }^{38}$ sharing similar entry criteria, all indicating superiority of lisdexamfetamine over placebo, ${ }^{23,40}$ as confirmed by preliminary number needed to treat and to harm data computed owing to the primary outcome "binge-eating days/week" of FDA-accounted placebo-controlled acute-treatment clinical trials. ${ }^{50}$ The encouraging results from a Phase III maintenance $\mathrm{RCT}^{32}$ (and its 12-week open-label safety extension study) would also be evaluated by the FDA for potential inclusion of these data to the current labeling of the drug.

From this perspective, we submit that the FDA approval of lisdexamfetamine as the first drug for the treatment of moderate-to-severe BED in adults should not be perceived as the mere consequence of "marketing strategy" taking advantage of a "diagnostic shift" or placebo-controlled "publication bias" related to existing drugs. Yet, we were unable to find any negative result trial or any eventual hint of potential "sponsorship bias" at the present time. 
Nonetheless, 1) a selection bias due to inadequate generation of randomized sequence, 2) inadequate concealment of allocation prior to assignment, 3) performance bias due to knowledge of the allocated interventions by participants and personnel during the studies, 4) attrition bias due to amount, nature, or handling of incomplete outcome data, 5) reporting bias due to selective outcome reporting, and 6) substantial lack of inclusion of AEs other than those requiring discontinuation of the trial may all have undermined the quality of the original studies in the accounted review and meta-analysis, ultimately further stressing out the need for additional, welldesigned, replication studies on the matter beyond those already undertaken by the drug manufacturer.

\section{Conclusion}

While the preliminary data suggest that lisdexamfetamine would have promising efficacy in CGI-S-defined response and in remission rates, as well as in reducing overall days with binge, weight and total Y-BOCS-BE scores, additional acute and especially maintenance-phase trials are warranted, whereas current evidence in support of safety of lisdexamfetamine in the acute treatment of moderate-to-severe BED in adults is tentative, but also unclear, thus urging for systematic controlled long-term assessment and monitoring. Since AEs and discontinuation rates were higher among patients receiving lisdexamfetamine rather than placebo, future independent research is urged to clarify the reliability of preliminary safety results made publicly available after sponsored trials.

\section{Author contributions}

All the authors contributed toward data analysis, drafting, and critically revising the paper and agree to be accountable for all aspects of the work.

\section{Disclosure}

The authors report no conflicts of interest in this work.

\section{References}

1. APA, editor. Diagnostic and Statistical Manual of Mental Disorders, Fourth Edition (DSM-IV). Washington (DC): APA; 1994.

2. APA. Diagnostic and Statistical Manual for Mental Disorders - Text Revision (DSM-IV-TR). Washington (DC): American Psychiatric Association; 2000.

3. Citrome L. A primer on binge eating disorder diagnosis and management. CNS Spectr. 2015;20(Suppl 1):41-51.

4. Thiede H, Hagan H, Campbell JV, et al. Prevalence and correlates of indirect sharing practices among young adult injection drug users in five U.S. cities. Drug Alcohol Depend. 2007;91(Suppl 1):S39-S47.

5. Kessler RC, Berglund PA, Chiu WT, et al. The prevalence and correlates of binge eating disorder in the World Health Organization World Mental Health Surveys. Biol Psychiatry. 2013;73(9):904-914.
6. Marques L, Alegria M, Becker AE, et al. Comparative prevalence, correlates of impairment, and service utilization for eating disorders across US ethnic groups: Implications for reducing ethnic disparities in health care access for eating disorders. Int J Eat Disord. 2011;44(5):412-420.

7. Bruce B, Wilfley D. Binge eating among the overweight population: a serious and prevalent problem. J Am Diet Assoc. 1996;96(1):58-61.

8. Spitzer RL, Yanovski S, Wadden T, et al. Binge eating disorder: its further validation in a multisite study. Int J Eat Disord. 1993;13(2):137-153.

9. APA, editor. Diagnostic and Statistical Manual of Mental Disorders, Fifth Edition (DSM-5). Washington (DC): APA; 2013.

10. Solmi F, Hotopf M, Hatch SL, Treasure J, Micali N. Eating disorders in a multi-ethnic inner-city UK sample: prevalence, comorbidity and service use. Soc Psychiatry Psychiatr Epidemiol. 2016;51(3):369-381.

11. Grilo CM, Ivezaj V, White MA. Evaluation of the DSM-5 severity indicator for binge eating disorder in a clinical sample. Behav Res Ther. 2015;71:110-114.

12. Marek RJ, Ben-Porath YS, Ashton K, Heinberg LJ. Impact of using DSM-5 criteria for diagnosing binge eating disorder in bariatric surgery candidates: change in prevalence rate, demographic characteristics, and scores on the Minnesota Multiphasic Personality Inventory-2 restructured form (MMPI-2-RF). Int J Eat Disord. 2014;47(5):553-557.

13. Trace SE, Thornton LM, Root TL, et al. Effects of reducing the frequency and duration criteria for binge eating on lifetime prevalence of bulimia nervosa and binge eating disorder: implications for DSM-5. Int J Eat Disord. 2012;45(4):531-536.

14. Herman BK, Safikhani S, Hengerer D, et al. The patient experience with DSM-5-defined binge eating disorder: characteristics, barriers to treatment, and implications for primary care physicians. Postgrad Med. 2014; 126(5):52-63.

15. Reas DL, Grilo CM. Pharmacological treatment of binge eating disorder: update review and synthesis. Expert Opin Pharmacother. 2015; 16(10):1463-1478.

16. Vancampfort D, Vanderlinden J, Pieters G, et al. [The importance of movement-directed interventions in the multidisciplinary treatment of binge eating disorder: an overview]. Tijdschr Psychiatr. 2012; 54(8):719-730.

17. Amianto F, Ottone L, Abbate Daga G, Fassino S. Binge-eating disorder diagnosis and treatment: a recap in front of DSM-5. BMC Psychiatry. 2015;15:70.

18. McElroy SL, Guerdjikova AI, Mori N, O’Melia AM. Pharmacological management of binge eating disorder: current and emerging treatment options. Ther Clin Risk Manag. 2012;8:219-241.

19. Berkman ND, Brownley KA, Peat CM, et al. Management and Outcomes of Binge-Eating Disorder. Rockville (MD): Agency for Healthcare Research and Quality (AHRQ); 2015.

20. Smith DG, Robbins TW. The neurobiological underpinnings of obesity and binge eating: a rationale for adopting the food addiction model. Biological Psychiatry. 2013;73(9):804-810.

21. Yager J. Binge eating disorder: the search for better treatments. Am J Psychiatry. 2008;165(1):4-6.

22. Guerdjikova AI, McElroy SL. Binge eating disorder pharmacotherapy clinical trails - who is left out? Eur Eat Disord Rev. 2009;17(2): 101-108.

23. McElroy SL, Guerdjikova AI, Mori N, Munoz MR, Keck PE. Overview of the treatment of binge eating disorder. CNS Spectr. 2015;20(6):546-556.

24. FDA. FDA expands uses of Vyvanse to treat binge-eating disorder. FDA News Release; 2015.

25. Moher D, Liberati A, Tetzlaff J, Altman DG; PRISMA Group. Preferred reporting items for systematic reviews and meta-analyses: the PRISMA statement. PLoS Med. 2009;6(7):e1000097.

26. Stroup DF, Berlin JA, Morton SC, et al. Meta-analysis of observational studies in epidemiology: a proposal for reporting. Meta-analysis of observational studies in epidemiology (MOOSE) group. Jama. 2000; 283(15):2008-2012. 
27. Striegel-Moore RH, Franko DL. Should binge eating disorder be included in the DSM-V? A critical review of the state of the evidence. Annu Rev Clin Psychol. 2008;4:305-324.

28. Qi X, Yang M, Ren W, et al. Find duplicates among the PubMed, EMBASE, and Cochrane Library Databases in systematic review. PloS One. 2013;8(8):e71838.

29. Cochrane Collaboration Group. Review Manager(RevMan) for Windows: Version 5.3. The Nordic Cochrane Centre, Copenhagen, Denmark. Available from: http://tech.cochrane.org/revman. 2015.

30. Deal LS, Wirth RJ, Gasior M, Herman BK, McElroy SL. Validation of the Yale-Brown obsessive compulsive scale modified for binge eating. Int J Eat Disord. 2015;48(7):994-1004.

31. Guy W. Clinical global impression scale. The ECDEU Assessment Manual for Psychopharmacology-Revised Volume DHEW Publ No ADM 76-338; 1976:218-222.

32. Hudson JI, et al. Vyvanse (lisdexamfetamine dimesylate) positive topline results in maintenance of efficacy study in adults with moderate to severe binge eating disorder [press release]; 2015 [July 22]. Available from: https://www.shire.com/newsroom/2015/july/vyvanse-positivetop-line-results-in-maintenance-of-efficacy-study-in-adults. Accessed March 1, 2016.

33. Smeeth L, Haines A, Shah E. Numbers needed to treat derived from metaanalyses - sometimes informative, usually misleading. Br Med J. 1999; 318(7197):1548.

34. Marx A, Bucher HC. Numbers needed to treat derived from metaanalysis: a word of caution. Evid Based Med. 2003;8(2):36-37.

35. Comprehensive meta-analysis ${ }^{\mathrm{TM}}$ version 3 [computer program]. 2014.

36. Higgins JP, Altman DG, Gotzsche PC, et al. The Cochrane Collaboration's tool for assessing risk of bias in randomised trials. BMJ. 2011; 343(7829):d5928.

37. Taylor S, Tweedie R. A non-parametric "trim and fill" method of assessing publication bias in meta-analysis. Denver: University of Colorado Health Sciences Center. 1998.

38. McElroy SL, Hudson JI, Mitchell JE, et al. Efficacy and safety of lisdexamfetamine for treatment of adults with moderate to severe binge-eating disorder: a randomized clinical trial. JAMA Psychiatry. 2015;72(3):235-246.
39. McElroy SL, Hudson J, Ferreira-Cornwell MC, Radewonuk J, Whitaker T, Gasior M. Lisdexamfetamine dimesylate for adults with moderate to severe binge eating disorder: results of two pivotal phase 3 randomized controlled trials. Neuropsychopharmacology. 2016;41(5):1251-1260.

40. Guerdjikova AI, Mori N, Casuto LS, McElroy SL. Novel pharmacologic treatment in acute binge eating disorder - role of lisdexamfetamine. Neuropsychiatr Dis Treat. 2016;12:833-841.

41. Agh T, Pawaskar M, Nagy B, Lachaine J, Voko Z. The cost effectiveness of lisdexamfetamine dimesylate for the treatment of binge eating disorder in the USA. Clin Drug Investig. 2016;36(4):305-312.

42. Rabiner DL. Stimulant prescription cautions: addressing misuse, diversion and malingering. Curr Psychiatry Rep. 2013;15(7):375.

43. Kroutil LA, Van Brunt DL, Herman-Stahl MA, Heller DC, Bray RM, Penne MA. Nonmedical use of prescription stimulants in the United States. Drug Alcohol Depend. 2006;84(2):135-143.

44. Lakhan SE, Kirchgessner A. Prescription stimulants in individuals with and without attention deficit hyperactivity disorder: misuse, cognitive impact, and adverse effects. Brain Behav. 2012;2(5):661-677.

45. Abenhaim L, Moride Y, Brenot F, et al. Appetite-suppressant drugs and the risk of primary pulmonary hypertension. $N$ Engl J Med. 1996; 335(9):609-616.

46. Klein-Schwartz W. Abuse and toxicity of methylphenidate. Curr Opin Pediatr. 2002;14(2):219-223.

47. Davey J, Turner RM, Clarke MJ, Higgins JP. Characteristics of metaanalyses and their component studies in the Cochrane Database of Systematic Reviews: a cross-sectional, descriptive analysis. BMC Med Res Methodol. 2011;11(1):1.

48. Kicinski M, Springate DA, Kontopantelis E. Publication bias in metaanalyses from the Cochrane Database of Systematic Reviews. Stat Med. 2015;34(20):2781-2793.

49. Sterne JA, Gavaghan D, Egger M. Publication and related bias in metaanalysis: power of statistical tests and prevalence in the literature. J Clin Epidemiol. 2000;53(11):1119-1129.

50. Citrome L. Lisdexamfetamine for binge eating disorder in adults: a systematic review of the efficacy and safety profile for this newly approved indication - what is the number needed to treat, number needed to harm and likelihood to be helped or harmed? Int J Clin Pract. 2015;69(4): 410-421.
Neuropsychiatric Disease and Treatment

\section{Publish your work in this journal}

Neuropsychiatric Disease and Treatment is an international, peerreviewed journal of clinical therapeutics and pharmacology focusing on concise rapid reporting of clinical or pre-clinical studies on a range of neuropsychiatric and neurological disorders. This journal is indexed on PubMed Central, the 'PsycINFO' database and CAS,

\section{Dovepress}

and is the official journal of The International Neuropsychiatric Association (INA). The manuscript management system is completely online and includes a very quick and fair peer-review system, which is all easy to use. Visit http://www.dovepress.com/testimonials.php to read real quotes from published authors. 